\title{
Baseline Low ALT Activity, a Marker of Sarcopenia and Frailty, is Associated with Increased Long-Term Mortality after COPD Exacerbations. Historic Prospective Cohort Study.
}

Nir Lasman

Sheba Medical Center at Tel Hashomer

Matan Shalom

Sheba Medical Center at Tel Hashomer

Natia Turpashvili

Sheba Medical Center at Tel Hashomer

Gal Goldhaber

Sheba Medical Center at Tel Hashomer

Yulia Lifshitz

Sheba Medical Center at Tel Hashomer

Eyal Leibowitz

Yoseftal medical center

Gideon Berger

Rambam Health Care Campus

Gilat Zalzman-Shenhav

Sheba Medical Center at Tel Hashomer

Adi Brom

Sheba Medical Center at Tel Hashomer

Dor Cohen

Sheba Medical Center at Tel Hashomer

Chen Avaki

Sheba Medical Center at Tel Hashomer

Gad Segal ( $\sim$ gad.segal@sheba.health.gov.il)

https://orcid.org/0000-0002-3851-3245

Research article

Keywords: ALT; COPD; Exacerbation; Frailty; Sarcopenia; Survival

Posted Date: May 5th, 2020 
DOI: https://doi.org/10.21203/rs.2.17787/v3

License: (c) (1) This work is licensed under a Creative Commons Attribution 4.0 International License. Read Full License 


\section{Abstract}

Background. COPD exacerbations have negative impact on patients' survival. Several risk factors for grave outcomes of such exacerbations have been descried. Muscle dysfunction and mass loss were shown to impact negatively on prognosis and survival. Low activity of the enzyme ALT (Alanine aminotransferase) in the blood is a known indicator for sarcopenia and frailty, however, no previous studies addressed the association of low ALT amongst patients hospitalized due to COPD exacerbation and longterm survival.

Methods. This is a historic prospective cohort study of patients hospitalized due to acute COPD exacerbation.

Results. Included were 232 consecutive COPD exacerbation patients. The median time of follow-up was 34.9 months (IQR 23.13 - 41.73 months). During this period 104 (44.8\%) patients died. All patients were grouped to quartiles according to blood ALT levels (after exclusion of cases considered to have hepatic tissue damage (ALT > $40 \mathrm{IU})$ ). The risk of long-term mortality increased, in a statistically significant manner, amongst patients with low ALT values: the median survival of patients with ALT < 11 IU was 18.5 months only while the median survival for the rest of the study group was not reached. For ALT < 11IU; $12-16 \mathrm{IU} ; 17-20 \mathrm{IU}$ and $>21 \mathrm{IU}$ the mortality rates were $69 \% ; 40.9 \% ; 36.3 \%$ and $25 \%$ respectively $(p<0.001$ for comparison of lower quartile with upper three quartiles). The crude hazard ratio for mortality amongst patients with ALT levels lower than $11 \mathrm{IU}$ was 2.37 (95\% Cl; $1.6-3.5)$. This increased risk of mortality remained significant after adjustment for age, weight, creatinine, albumin concentration and cardiovascular diseases $(\mathrm{HR}=1.83 ; 95 \% \mathrm{Cl} 1.08-3.1, \mathrm{p}<0.05)$.

Conclusions. Low ALT values, a biomarker of sarcopenia and frailty, are associated with poor long-term survival amongst patients hospitalized due to COPD exacerbation.

\section{Background}

Chronic obstructive pulmonary disease (COPD) is a highly prevalent disease worldwide. Acute COPD exacerbations have dismal results, both in high patients' mortality (ranging in different publications from over $5 \%$ to over $18 \%$ in-hospital mortality during exacerbation $(1,2))$ and in extremely large health expenses (1-6). Several risk factors for grave outcomes of COPD exacerbations have been described: Sakamoto et al. (6) performed multivariable logistic regression analysis of over 3,000 COPD patients and found that older age and lower body mass index, amongst other patients' characteristics, are significantly associated with increased risk of in-hospital mortality. Serra-Picamal et al., in a big-data analysis of 17,555 COPD patients, identified early re-hospitalizations as another risk factor for mortality after hospitalization due to COPD exacerbation (7).

Survival and COPD mortality are influenced by both dysfunction and impaired muscle mass (sarcopenia) $(8,9)$. Additionally, COPD exacerbations also rapidly induce loss of muscle mass and function through the activation of several biological pathways and systems. Sarcopenia (decreased muscle mass 
associated with increased risk of hospitalizations, falls and mortality) and frailty (increased tendency to succumb to morbidities, often associated with sarcopenia), two overlapping syndromes, defined as causing increased mortality and frequent hospital admissions, are prime candidates for having a negative impact on COPD exacerbations' outcomes. In a review published back in 2005, Morley et al. (10) stated that COPD is one of the chronic diseases particularly associated with frailty. Vellas et al. (11) relate to COPD as a causative agent for decreased muscle mass, in turn becoming a risk factor for COPD patients' mortality. In their review regarding "Sarcopenia and frailty in chronic respiratory disease", Bone et al. (12) declare the association between frailty and poor outcomes amongst COPD patients. Maddocks et al. (13) found that frailty affect $25 \%$ of all COPD patients and that it is a significant factor for pulmonary rehabilitation failure. Nevertheless, they state that pulmonary rehabilitation programs are beneficial for frail, COPD patients, potentially reversing frailty characteristics even with a short-term rehabilitation program.

Low ALT activity in the peripheral blood is associated with low, total-body, muscle mass. This is specifically accurate when there is no spillage of ALT from hepatocytes to the peripheral blood, such as in cases of hepatitis. Therefore, in the current study, as done in previous studies, patients with higher than normal ALT values were excluded from analysis. Previous studies have demonstrated a significant correlation between ALT values and muscle mass, as demonstrated by computed tomography measurements (14) and between low ALT and frailty, as appreciated by the FRAIL questionnaire (15). Other studies have showed that low ALT levels are associated with long term all-cause mortality in the elderly population (16-19). Sarcopenia and the resultant frailty, represented by low activity of the enzyme ALT (SGPT) in the peripheral blood, were shown to be associated with shortened survival and other poor clinical outcomes, in various patients' populations and clinical scenarios. It is postulated that this association rise from the fact that low ALT levels are the result of sarcopenia and the resultant frailty is the cause for shortening of life span in low-ALT populations (20-23).

However, there are no previous studies that examined the relationship between low ALT values and mortality amongst hospitalized patients with COPD exacerbation. The aim of the current study was to assess the association between ALT and clinical outcomes.

\section{Methods}

\section{Patient selection}

We executed a historic, prospective cohort study of COPD patients hospitalized to a single department of internal medicine, due to COPD exacerbation as their main diagnosis. The inclusion criteria were: a. age over 18 years, b. diagnosis of COPD exacerbation (ICD code 496) on discharge, c. available ALT blood levels, within the normal limits (lower than $40 \mathrm{IU} / \mathrm{L}$ ) during the 3 months prior to admission (in-hospital ALT levels, normally ranging from 7 to $45 \mathrm{IU} / \mathrm{L}$, were tested using a standard Beckman Coulter ${ }^{\circledR}$. Quantitative determination of ALT levels was used by applying kinetic UV tests. In order to assure maximal catalytic activity of the ALT from the blood samples, test tubes were supplemented with 
activated pyridoxal phosphate (P-5-P), serving as an essential co-factor for ALT catalytic activity. There are no significant normal-range differences between in- and out-of-hospital ALT measurement methods). Over $95 \%$ of ALT baseline values were available from in-hospital measurements while a small minority of ALT values were extracted from pre-hospitalization records. After study approval by an institutional ethics review board, we excluded patients with ALT levels greater than $40 \mathrm{IU} / \mathrm{L}$, supposed to have hepatic tissue damage of any cause. We extracted patients' details from the computerized records, including epidemiologic characteristics, background diagnoses, long-term medications, laboratory parameters, short- and long-term clinical outcomes. Data extraction from patients' computerized records was made by two researchers who went through all "free text" contents of these records. Mortality data was extracted from the Israeli national death certificates registry. The Norton scale, a score that is used by nurses around the world since the early 1960 s to assess the risk of pressure ulcers upon admission and during hospitalization, was extracted too from the medical record. It includes five domains, each is a fundamental aspect of health in the elderly: physical condition, mental condition, activity, mobility, and incontinence (24).

\section{Statistical methods}

In order to assess the association between ALT levels and dichotomous variables we calculated the sample size, as appropriate for medium strength associations. In order to determine such association, we needed a sample size greater than 192 patients (for $1 \%$ significance and $80 \%$ power). The cohort size was determined using G Power software (25).

We assessed the distribution of continuous variables using histograms and Q-Q plots. We described continuous variables with normal distribution by means and standard deviations. Continuous variables that did not have normal distribution were described using median and IQR (Inter-quartile range). We described categorical variables using frequency and percentages. ALT level was divided into two categories using the lower quartile as a cut off value for categorization. We assessed the association between ALT category and continuous variables using t-tests or Mann-Whitney tests. We assessed the association with categorical variables using the chi-square tests or the exact fisher's test. Kaplan-Meier curve was used to describe survival during the follow up period and log-rank test was used to compare between ALT categories. Univariate Cox regression was used to study the association between the various parameters and mortality. We used Cox regression to assess the association between ALT category while controlling for possible confounders. Statistical analysis was done using the SPSS software (IBM Corp., NY USA). All statistical tests were two-sided. We considered as significant only $p$ values lower than 0.05 .

\section{Results}

We included in this study 232 consecutive COPD patients, hospitalized due to COPD exacerbation in one internal-medicine department in a large, tertiary hospital, over a period of 38 months - from January 2015 to February 2018. After approval by an IRB, we extracted patients' data from their computerized 
medical records. The mean age was $76.06 \pm 10.7$ years, median Norton score was 15.7 (IQR 13-19), median ALT level was $16.5 \mathrm{IU} / \mathrm{L}$ (IQR 11-20), median creatinine concentration was $1.15 \mathrm{mg} / \mathrm{dL}$ (IQR $0.74-$ 1.35), and the mean albumin concentration was $3.6 \pm 0.47 \mathrm{~g} / \mathrm{dL}$. Within our cohort, $74.6 \%$ had cardiovascular diseases, $59.9 \%$ had metabolic diseases, $35.3 \%$ had diabetes, $25.4 \%$ had neurological diseases, and $24.1 \%$ suffered from hematological or hemato-oncological diseases. Regarding background medical treatments, $19.4 \%$ were treated by systemic steroids, $32.8 \%$ took anti-diabetic medications, and $84.1 \%$ had cardiovascular medications, $26.3 \%$ treated by anticoagulants and $67.7 \%$ were using inhalers regularly. Patients' characteristics, according to their baseline ALT values (lower quartile $(<11 \mathrm{IU} / \mathrm{L}) \mathrm{vs}$. upper three quartiles (11 IU/L < ALT < $40 \mathrm{IU} / \mathrm{L})$ ), are described in table 1. The median time of follow-up was 34.9 months (IQR 23.13 - 41.73). During this period $104(44.8 \%)$ patients died. Figure 1 describe the flow of patients from recruitment to analysis and table 2 describe patient characteristics found to be associated with increased risk for mortality amongst our study cohort.

We divided all patients to quartiles according to their blood ALT levels (after exclusion of cases considered to have hepatic tissue damage (ALT > $40 \mathrm{IU})$ ). The risk of long-term mortality increased, in a statistically significant manner, amongst patients with low ALT values: the median survival for patients with ALT < 11 IU was 18.5 months only while the median survival for the rest of the study group was not reached. For ALT < 11IU; 12-16IU; 17-20IU and >21IU the mortality rates were $69 \% ; 40.9 \% ; 36.3 \%$ and $25 \%$ respectively ( $p<0.001$ for comparison of lower quartile with upper three quartiles; figure 2$)$.

The crude hazard ratio for mortality amongst patients with ALT levels lower than 11lU was 2.37 (95\% Cl; $1.6-3.5)$. This increased risk of mortality remained significant after controlling for age, weight, Norton score, creatinine, albumin concentration and cardiovascular diseases $(\mathrm{HR}=1.83 ; 95 \% \mathrm{Cl} 1.08-3.1, \mathrm{p}=$ 0.025). Table 3 describe the multivariate Cox regression for mortality in our study cohort.

\section{Discussion}

As stated earlier, ALT is a biomarker for sarcopenia and frailty $(14,15,23)$. In our study, we have showed that low ALT values, are associated with poor long-term survival amongst patients hospitalized due to COPD exacerbation. Of note, our results remained significant after controlling for parameters associated with frailty, i.e. Norton scale, albumin, etc.

Appreciation of sarcopenia and frailty enables a summation of patients' characteristics which are associated with his or her risk for subsequent morbidity and mortality. Addressing sarcopenia and frailty in quantitative measures should, therefore, better the prognostication process for many patients' populations. COPD patients should not be an outlier for the aforementioned declaration: these patients should be routinely evaluated for sarcopenia and frailty, and treatment strategies must take into account these patient's characteristics. Routine assessment for sarcopenia and frailty can be done by simple ALT measurements or by phenotypic tests such as hand grip strength test of the "up and go" test.

Current literature promotes personalized medicine. However, most cases of personalized medicine are actually dealing with precise medicine: diagnostic approaches become more focused on disease and 
pathology rather than on the person / patient as a whole. We believe that thorough evaluation of sarcopenia and frailty should be considered as true personalized medicine.

In the current study we show that similarly to other patients' groups, COPD patients can also be classified as sarcopenic and frail, according to their baseline ALT values. ALT measurements are a part of routine blood tests and are available worldwide for any hospitalized patient. Acknowledgment of low ALT values during COPD exacerbation could aid the attending physician in taking better clinical / therapeutic decisions (duration and route of administration of steroids, duration and type of antibiotics, promoting physiotherapy sessions and rehabilitation programs prior to patient's discharge to the community etc.).

ALT measurement should not replace other, well established methods for sarcopenia and frailty appreciation. Nevertheless, most such evaluation tools were developed and are being used in the realm of geriatric medicine. The results of this study are applicable to the general population of COPD patients admitted to internal-medicine departments. For these reasons, we suggest that ALT values would be flagged, and diagnoses of sarcopenia and frailty should be highlighted in the discharge documents of COPD patients.

\section{Limitations}

In the study we performed a retrospective analysis and therefore, not all confounding parameters could be addressed (such as $\mathrm{BMI}$ and $\mathrm{FEV}_{1}$ values). Moreover, while demonstrating significant statistical associations between ALT and mortality, causality could not be inferred. We performed the study on patients admitted to a single internal medicine department that might not correlate perfectly to all COPD patients that are admitted to hospitals nationally.

\section{Conclusion}

Low ALT values are an independent marker for shortened survival of COPD patients after being hospitalized due to COPD exacerbation. Being a marker for sarcopenia and frailty, low ALT values in these patients should prompt more aggressive therapeutic efforts on behalf of the attending physician. Future, prospective, interventional studies should aim at evaluation of different therapies and rehabilitation programs, concentrated at the sarcopenic and frail COPD patients.

\section{Declarations}

\section{Ethics approval and consent to participate}

This study was initiated only after approval by the institutional review board at the Chaim Sheba medical center. Approval number 5779-18-SMC. Since this was a retrospective study, consent was not relevant.

$\underline{\text { Consent to publish }}$

All authors gave their consent for publication of the completed manuscript. 
Availability of data and materials

All data is available with the authors for further study questions.

Competing interests

All authors declare they have no competing interests regarding this study and manuscript.

Funding

No external funding was given to this study. No funding to report.

Authors' contributions

Study design and planning: LN; SM; TN; GG; LY; LE; BG; ZSG; BA; CD; AC; SG. Data collection: LN; SM; TN; GG; SG. Data analysis: LN; SM; TN; GG; LY; LE; BG; ZSG; BA; CD; AC; SG. Manuscript writing and proofs: LN; SM; TN; GG; LY; LE; BG; ZSG; BA; CD; AC; SG. All authors have read and approved the manuscript.

\section{Acknowledgements}

NA

Authors' information

Gad Segal, MD. Internal medicine T, Chaim Sheba medical center, Tel Hashomer, $2^{\text {nd }}$ Sheba road, Ramat Gan. Israel. Gad.segal@sheba.health.gov.il.+972-52-6669580.

\section{Abbreviations}

ALT; Alanine amino-transferase. SGPT; Serum glutamic pyruvic transaminase. COPD; Chronic, obstructive pulmonary disease. Cl; Confidence interval. IU; International units. IQR; Inter-quartile range. Q-Q Plot; Quantile-quantile plot.

\section{References}

1. Laribi S, Keijzers G, van Meer O, Klim S, Motiejunaite J, Kuan W Sen, et al. Epidemiology of patients presenting with dyspnea to emergency departments in Europe and the Asia-Pacific region. Eur $\mathrm{J}$ Emerg Med. 2018 Aug;1.

2. Brown H, Dodic S, Goh SS, Green C, Wang W, Kaul S, et al. Factors associated with hospital mortality in critically ill patients with exacerbation of COPD. Int J Chron Obstruct Pulmon Dis. 2018 Aug;Volume 13:2361-6.

3. Barrecheguren M, González C, Miravitlles M. What have we learned from observational studies and clinical trials of mild to moderate COPD? Respir Res. 2018 Dec;19(1):177. 
4. Ryrs $\varnothing$ CK, Godtfredsen NS, Kofod LM, Lavesen M, Mogensen L, Tobberup R, et al. Lower mortality after early supervised pulmonary rehabilitation following COPD-exacerbations: a systematic review and meta-analysis. BMC Pulm Med. 2018 Dec;18(1):154.

5. Takechi Y. [Intervention for COPD Exacerbation - How To Prevent and Treat COPD Exacerbation ?]. Gan To Kagaku Ryoho. 2016 Dec;43(Suppl 1):61-3.

6. Sakamoto Y, Yamauchi Y, Yasunaga H, Takeshima H, Hasegawa W, Jo T, et al. Development of a nomogram for predicting in-hospital mortality of patients with exacerbation of chronic obstructive pulmonary disease. Int J Chron Obstruct Pulmon Dis. 2017 May;Volume 12:1605-11.

7. Serra-Picamal X, Roman R, Escarrabill J, García-Altés A, Argimón JM, Soler N, et al. Hospitalizations due to exacerbations of COPD: A big data perspective. Respir Med. 2018 Jan;

8. Shrikrishna D, Patel M, Tanner RJ, Seymour JM, Connolly BA, Puthucheary ZA, et al. Quadriceps wasting and physical inactivity in patients with COPD. Eur Respir J. 2012 Nov;40(5):1115-22.

9. Swallow EB, Reyes D, Hopkinson NS, Man WD-C, Porcher R, Cetti EJ, et al. Quadriceps strength predicts mortality in patients with moderate to severe chronic obstructive pulmonary disease. Thorax. 2007 Feb;62(2):115-20.

10. Morley JE, Kim MJ, Haren MT, Kevorkian R, Banks WA. Frailty and the aging male. Aging Male. 2005 Jan;8(3-4):135-40.

11. Vellas B, Fielding R, Bhasin S, Cerreta F, Goodpaster B, Guralnik JM, et al. Sarcopenia Trials in Specific Diseases: Report by the International Conference on Frailty and Sarcopenia Research Task Force. J frailty aging. 2016;5(4):194-200.

12. Bone AE, Hepgul N, Kon S, Maddocks M. Sarcopenia and frailty in chronic respiratory disease. Chron Respir Dis. 2017 Feb;14(1):85-99.

13. Maddocks M, Kon SSC, Canavan JL, Jones SE, Nolan CM, Labey A, et al. Physical frailty and pulmonary rehabilitation in COPD: a prospective cohort study. Thorax. 2016;71(11):988-95.

14. Portal D, Hofstetter L, Eshed I, Dan Lantsman C, Sella T, Urban D, et al. L3 skeletal muscle index (L3SMI) is a surrogate marker of sarcopenia and frailty in non-small cell lung cancer patients. Cancer Manag Res. 2019 Apr;Volume 11:2579-88.

15. Irina G, Refaela C, Adi B, Avia D, Liron H, Chen A, et al. Low Blood ALT Activity and High FRAIL Questionnaire Scores Correlate with Increased Mortality and with Each Other. A Prospective Study in the Internal Medicine Department. J Clin Med. 2018 Oct;7(11):386.

16. Elinav E, Ackerman Z, Maaravi Y, Ben-Dov IZ, Ein-Mor E, Stessman J. Low alanine aminotransferase activity in older people is associated with greater long-term mortality. J Am Geriatr Soc. 2006 Nov;54(11):1719-24.

17. Koehler EM, Sanna D, Hansen BE, van Rooij FJ, Heeringa J, Hofman A, et al. Serum liver enzymes are associated with all-cause mortality in an elderly population. Liver Int. 2014 Feb;34(2):296-304.

18. Le Couteur DG, Blyth FM, Creasey HM, Handelsman DJ, Naganathan V, Sambrook PN, et al. The association of alanine transaminase with aging, frailty, and mortality. J Gerontol A Biol Sci Med Sci. $2010 \mathrm{Jul} ; 65(7): 712-7$. 
19. Vespasiani-Gentilucci U, De Vincentis A, Ferrucci L, Bandinelli S, Antonelli Incalzi R, Picardi A. Low Alanine Aminotransferase Levels in the Elderly Population: Frailty, Disability, Sarcopenia, and Reduced Survival. J Gerontol A Biol Sci Med Sci. 2018 Jun;73(7):925-30.

20. Ramati E, Israel A, Tal Kessler, Petz-Sinuani N, Sela B-A, Idan Goren, et al. [Low ALT activity amongst patients hospitalized in internal medicine wards is a widespread phenomenon associated with low vitamin B6 levels in their blood]. Harefuah. 2015 Feb;154(2):89-93, 137.

21. Ramaty E, Maor E, Peltz-Sinvani N, Brom A, Grinfeld A, Kivity S, et al. Low ALT blood levels predict long-term all-cause mortality among adults. A historical prospective cohort study. Eur J Intern Med. 2014 Dec;25(10):919-21.

22. Peltz-Sinvani N, Klempfner R, Ramaty E, Sela BA, Goldenberg I, Segal G. Low ALT Levels Independently Associated with 22-Year All-Cause Mortality Among Coronary Heart Disease Patients. J Gen Intern Med. 2016;31(2):209-14.

23. Gringauz I, Weismann J, Justo D, Adunsky A, Segal G. Alanine aminotransferase blood levels and rehabilitation outcome in older adults following hip fracture surgery. Int J Rehabil Res. 2018 Mar;41(1):41-6.

24. Norton D. Calculating the risk: reflections on the Norton Scale. Decubitus. 1989 Aug;2(3):24-31.

25. Erdfelder E, Faul F, Buchner A. GPOWER: A general power analysis program. Behav Res Methods, Instruments, Comput. 1996 Mar;28(1):1-11.

\section{Tables}

Table 01. Patients' characteristics according to baseline ALT levels 


\begin{tabular}{|c|c|c|c|}
\hline & $\mathrm{ALT}<11 \mathrm{IU} / \mathrm{L}$ & $11 \mathrm{IU} / \mathrm{L} \leq \mathrm{ALT} \leq 40 \mathrm{IU} / \mathrm{L}$ & $P$ value \\
\hline \multicolumn{4}{|l|}{ Patients' demographics } \\
\hline Age (years \pm SD) & $78.8 \pm 9$ & $74.8 \pm 11.2$ & 0.008 \\
\hline Gender - males (n (\%)) & $39(54.9)$ & $77(47.8)$ & 0.32 \\
\hline Norton score (number, IQR) & $14.93(13-17)$ & $16.06(13.5-19)$ & 0.002 \\
\hline \multicolumn{4}{|l|}{ Background diagnoses (groups) } \\
\hline Cancer (n (\%)) & $20(28.2)$ & $36(22.4)$ & 0.34 \\
\hline Dementia (n (\%)) & $3(4.2)$ & $7(4.3)$ & 0.96 \\
\hline Diabetes (n (\%)) & $33(46.5)$ & $49(30)$ & 0.018 \\
\hline Cardiovascular (n (\%)) & $54(76.1)$ & $119(73.9)$ & 0.73 \\
\hline Metabolic (n (\%)) & $47(66.2)$ & $92(57.1)$ & 0.19 \\
\hline Neurologic (n (\%)) & $17(23.9)$ & $42(26.1)$ & 0.73 \\
\hline Endocrine (n (\%)) & $12(16.9)$ & $21(13.0)$ & 0.49 \\
\hline \multicolumn{4}{|l|}{ Medications (groups) } \\
\hline Steroids (n (\%)) & $17(23.9)$ & $28(17.4)$ & 0.24 \\
\hline Anticoagulation (n (\%)) & $27(38)$ & $34(21.1)$ & 0.007 \\
\hline Inhalers/Inhalation (n (\%)) & $46(64.8)$ & $111(68.9)$ & 0.53 \\
\hline Anti-diabetic (n (\%)) & $28(39.4)$ & $48(29.8)$ & 0.15 \\
\hline Cardiovascular (n (\%)) & $62(87.3)$ & $133(82.6)$ & 0.37 \\
\hline Metabolic (n (\%)) & $17(23.9)$ & $33(20.5)$ & 0.56 \\
\hline Neurologic (n (\%)) & $33(46.5)$ & $62(36.5)$ & 0.25 \\
\hline \multicolumn{4}{|l|}{ Laboratory parameters } \\
\hline Albumin $(\mathrm{g} / \mathrm{dL} \pm \mathrm{SD})$ & $3.4 \pm 0.45$ & $3.7 \pm 0.45$ & $<0.001$ \\
\hline Creatinine (mg/dL, (IQR)) & $1.32(0.93-1.53)$ & $1.07(0.68-1.29)$ & $<0.001$ \\
\hline \multicolumn{4}{|l|}{ Clinical outcomes } \\
\hline Mortality (n (\%)) & $49(69.0)$ & $55(34.2)$ & \multirow[t]{2}{*}{$<0.001$} \\
\hline Survival time - median (months) & 18.5 & Median not reached & \\
\hline
\end{tabular}

Table 02. Patients' characteristics according to survival - univariate analysis.

\begin{tabular}{|l|c|c|c|c|}
\hline atients' characteristics & Alive & Dead & Hazard ratio (95\% CI) & P Value \\
\hline ye (years \pm SD) & $72.89 \pm 10.8$ & $79.96 \pm 9.2$ & $1.06(1.04-1.08)$ & $<0.001$ \\
\hline orton score (number, (IQR)) & $16.84(15-19)$ & $14.33(11.25-17)$ & $0.88(0.84-0.92)$ & $<0.001$ \\
\hline lbumin (mg/dL \pm SD) & $3.69 \pm 0.47$ & $3.48 \pm 0.45$ & $0.48(0.34-0.7)$ & $<0.001$ \\
\hline reatinine (mg/dL, (IQR)) & $1.03(0.69-1.21)$ & $1.29(0.84-1.62)$ & $1.57(1.23-2)$ & $<0.001$ \\
\hline LT (IU/L, IQR) & $18.71(13-23.75)$ & $13.77(9-17)$ & $0.93(0.9-0.96)$ & $<0.001$ \\
\hline LT < 11 IU/L (n (\%)) & $22(17.2 \%)$ & $49(47.1 \%)$ & $2.48(1.67-3.66)$ & $<0.001$ \\
\hline ardiovascular disease (n (\%)) & $89(69.5)$ & $84(80.8)$ & $1.8(1.1-2.93)$ & 0.019 \\
\hline ardiovascular medications (n (\%)) & $101(78.9)$ & $94(90.4)$ & $2.09(1.09-4.0)$ & 0.027 \\
\hline eurologic medications (n (\%)) & $41(32.0)$ & $54(51.9)$ & $1.94(1.31-2.86)$ & 0.001 \\
\hline nticoagulation medications (n (\%)) & $29(18.8)$ & $37(35.6)$ & $1.73(1.15-2.57)$ & 0.008 \\
\hline
\end{tabular}


Table 03. Multivariate Cox regression for mortality in our study cohort.

\begin{tabular}{|l|c|c|}
\hline atients' characteristics & Hazard ratio $(95 \% \mathrm{CI})$ & P value \\
\hline jwer quartile of ALT $(<11 \mathrm{IU} / \mathrm{L})$ & $1.83(1.08-3.1)$ & 0.025 \\
\hline ye (years) & $1.02(0.99-1.05)$ & 0.110 \\
\hline 'eight (kilograms) & $0.98(0.97-0.99)$ & 0.02 \\
\hline orton score $($ number) & $0.92(0.86-0.98)$ & 0.016 \\
\hline reatinine value $(\mathrm{mg} / \mathrm{dl})$ & $1.79(1.14-2.81)$ & 0.011 \\
\hline bumin concentration $(\mathrm{mg} / \mathrm{dl})$ & $0.67(0.41-1.18)$ & 0.178 \\
\hline ardiovascular disease & $1.56(0.83-2.94)$ & 0.167 \\
\hline
\end{tabular}

\section{Figures}

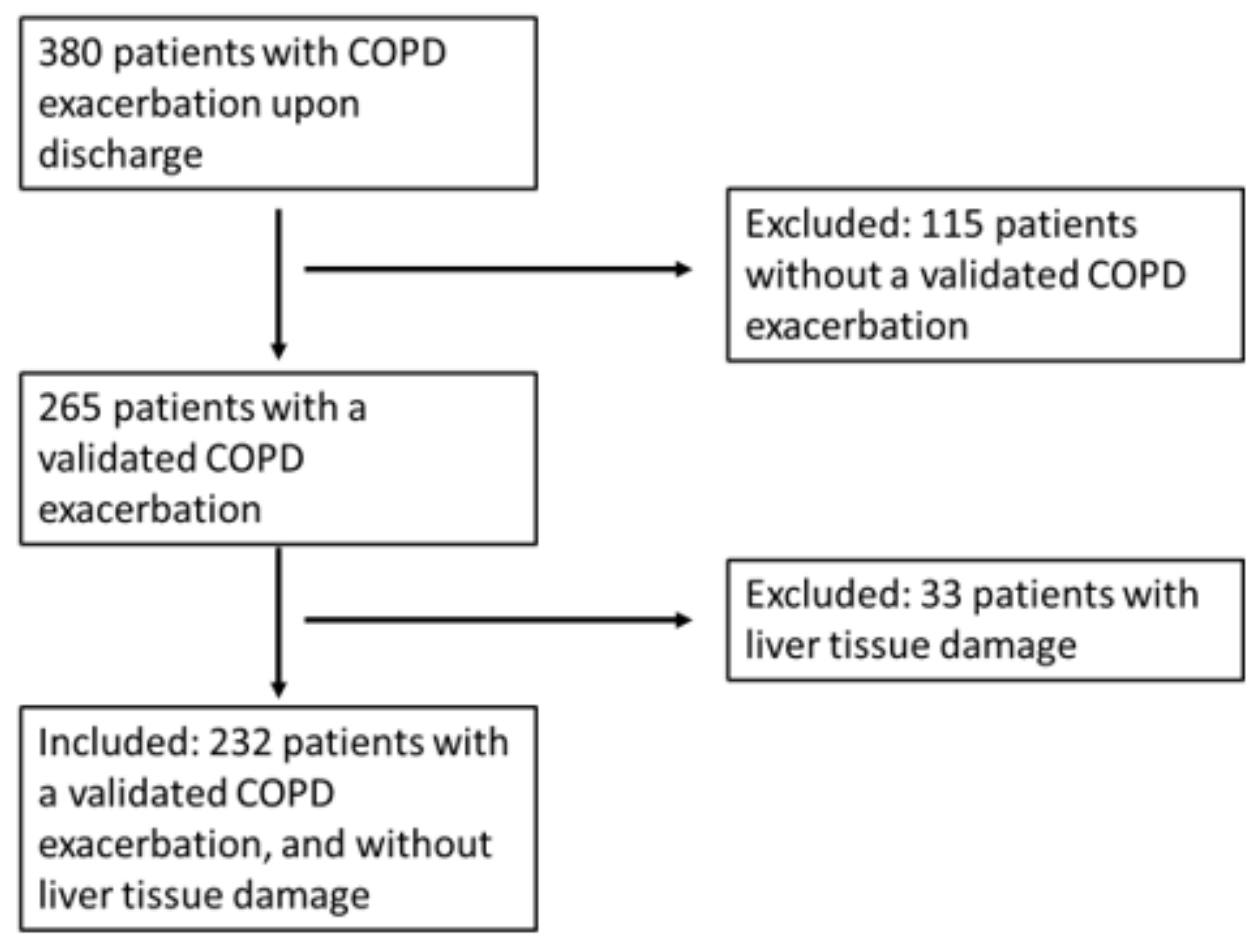

Figure 1

Consort diagram of patients' flow in the current study. 


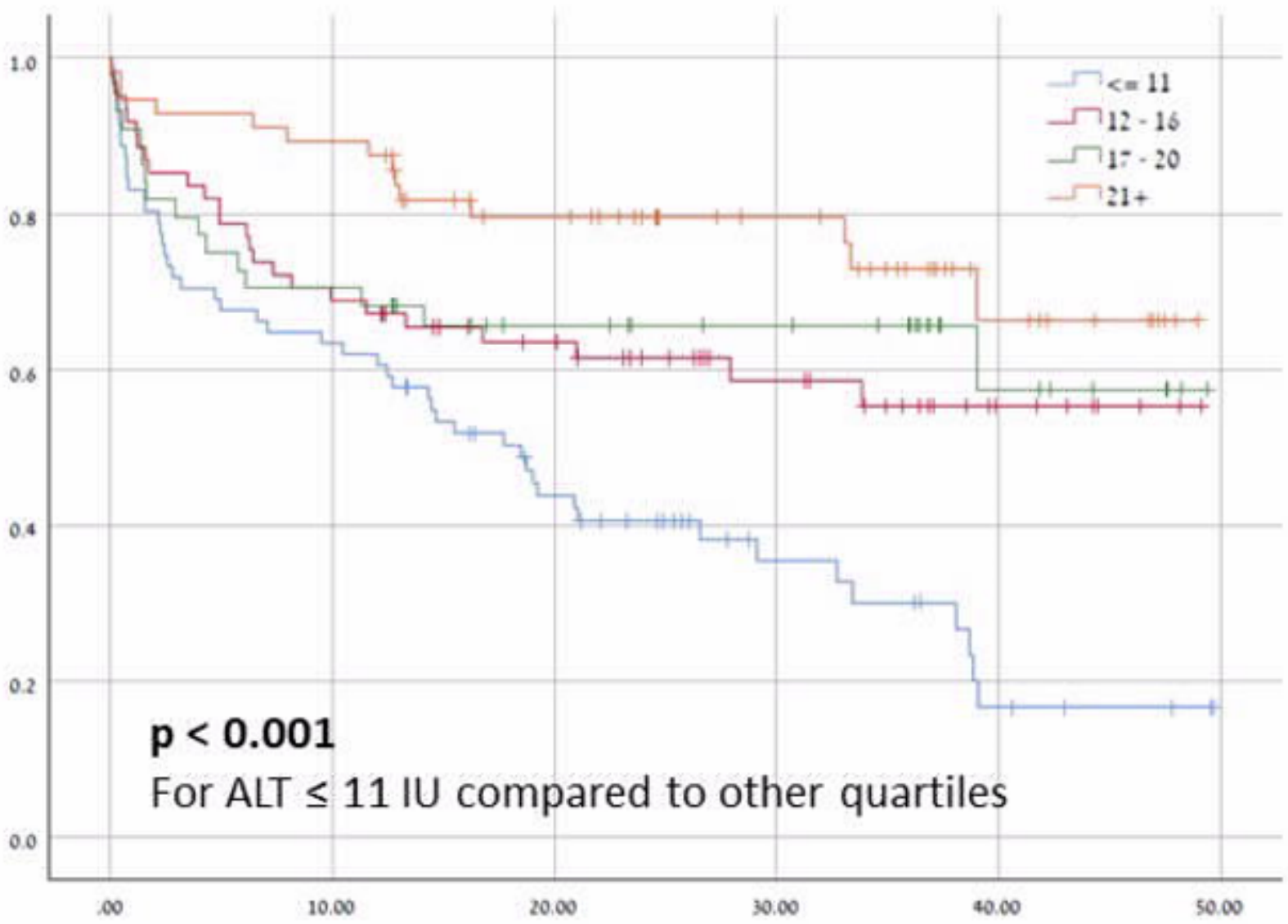

Figure 2

Survival (months) of COPD patients according to baseline ALT values 